\title{
The Magnet Measurement Facility for the Advanced Photon Source
}

OCT 191933

\section{OSTI}

Abstract-A magnet measurement facllity has been developed to measure the prototype and productlon magnets for the Advance Photon Source. The measurement facllity is semi-automatle In measurement control and data analysis. One dipole system and three rotallng coll measurement systems for quadrupole and sextupole magnets and corresponding probe colls are described.

\section{INTRODUCTION}

The 7-GeV Advance Photon Source (APS), which is under construction at Argonne National Laboratory (ANL.), is a national facility declicated to providing highly-brilliant synchrotron radiation beams [1]. The magnet system for the APS requires more than 1000 conventional resistive magnets for the storage ring (SR), injector synchrotron (IS), positron accumulator ring (PAR), and beam transport lines. Main characterisitics of the magnets, except those for the beam transports, are listed in Table 1. The SR magnets in particular

TABLE 1

MaIN Characteristics OF THE APS RING MAGNETS

\begin{tabular}{ccccc}
\hline Magnet & $\begin{array}{c}\text { Magneti } \\
\text { Length }(\mathrm{m})\end{array}$ & $\begin{array}{c}\text { Pole gap or } \\
\text { Bore Dia. }(\mathrm{mm})\end{array}$ & $\begin{array}{c}\text { Field } \\
\text { Strength }\end{array}$ & $\begin{array}{c}\text { No. of } \\
\text { Magnets }\end{array}$ \\
\hline SR Dipole & 3.06 & 60.00 & $0.599 \mathrm{~T}$ & 80 \\
SR Quad & $.5 / .6 / .8$ & 80.00 & $18.9 \mathrm{~T} / \mathrm{m}^{2}$ & 400 \\
SR Sext & 0.24 & 98.00 & $490 \mathrm{~T} / \mathrm{m}^{2}$ & 280 \\
& & & & \\
IS Dipole & 3.10 & 40.00 & $0.696 \mathrm{~T}$ & 68 \\
IS Quad & 0.60 & 56.56 & $13.9 \mathrm{~T} / \mathrm{m}^{2}$ & 80 \\
IS Sext & 0.20 & 70.01 & $130 \mathrm{~T} / \mathrm{m}^{2}$ & 68 \\
& & & & \\
PAR Dipole & 0.80 & 45.00 & $1.47 \mathrm{~T}$ & 8 \\
PAR Quad & 0.25 & 120.00 & $4 \mathrm{~T} / \mathrm{m}$ & 16 \\
PAR Sext & 0.20 & 130.00 & $10 \mathrm{~T} / \mathrm{m}$ & 10 \\
\hline
\end{tabular}

are required to measure and evaluate the field strength and field quality to a few parts in $10^{-4}$ to verify the tolerance requirements. Equally important parameters to be measured are the magnetic axis and roll angle, with respect to one or two fiducials located on top of a magnet, within tolerances of $60 \mu \mathrm{m}$ and $0.3 \mathrm{mrad}$, respectively.

Manuscript received September 20, 1993. This work was supported by the U.S. Department of Energy, Office of Basic Energy Sciences, under Contract No. W-31-109-ENG-38.
In order to meet the project schedule and required tolerances of the magnet parameters, a magnet measurement facility has been developed. The facility includes a dipole measurement system, three rotating coil systems for quadrupole (quad) and sextupole (sext) magnets, Hall-probe field mapping, and various types of probe coils [2]. The rotating coil systems are located in a temperature-conirolled area. The measurement systems have been extensively tested in parallel with the development of various prototype magnets. A typical production measurement takes less than four hours. The measured data for the production magnets are being used for the quality control of the magnet fabrication procedures and the correction of some magnets with unacceptable tolerances.

\section{DiPOLE MEASUREMENT SYSTEM}

A block diagram of the mechanical hardware assembly for the SR dipole measurement is shown in Fig. 1. The production dipcle magnets are measured comparatively with respect to a reíerence dipole. Two sets of curved integral coils are connected in series, one fixed in the reference and one for a testing production dipole. The field integral is measured by ramping the excitation curreni of the dipole up and down, while the field shane is measured by moving the coil set in a transverse direction. The transverse positons of the integral coils in the production dipole are controlled by three positioning tables which are attached to a 5-m-long granite table. Since the pole gap of the "C-type" SR dipole is accessible from one side of the magnet, this measurement procedure is fully automated. For measurement of an IS dipole which has an "H-type" cross section, the probe coils are installed and removed manually through one end of the magnet.

A set of integral coils consists of two coils: a $3.5-\mathrm{m}$-long coil for full integral and a 1.5-m-long coil for 2-D "body" field measurements. The two coils in the reference dipole are used as the bucking coils for the integral and 2-D field measurements. The probe coils are made by placing two layers of flat printed curcuit (PC) coils on a honeycombstructured G-10 plate according to the curvature of the magnet, and then connected in series. A PC coil is $0.5 \mathrm{~m}$ long with an average width of $6.8 \mathrm{~mm}$, height of $3.3 \mathrm{~mm}$, and 170 turns.

A 3-D field mapping unit with a calibrated Hall probe also measures detailed end-field and 2-D field-.. tape for the prototype and reference magnets. The Hall probe mapping 


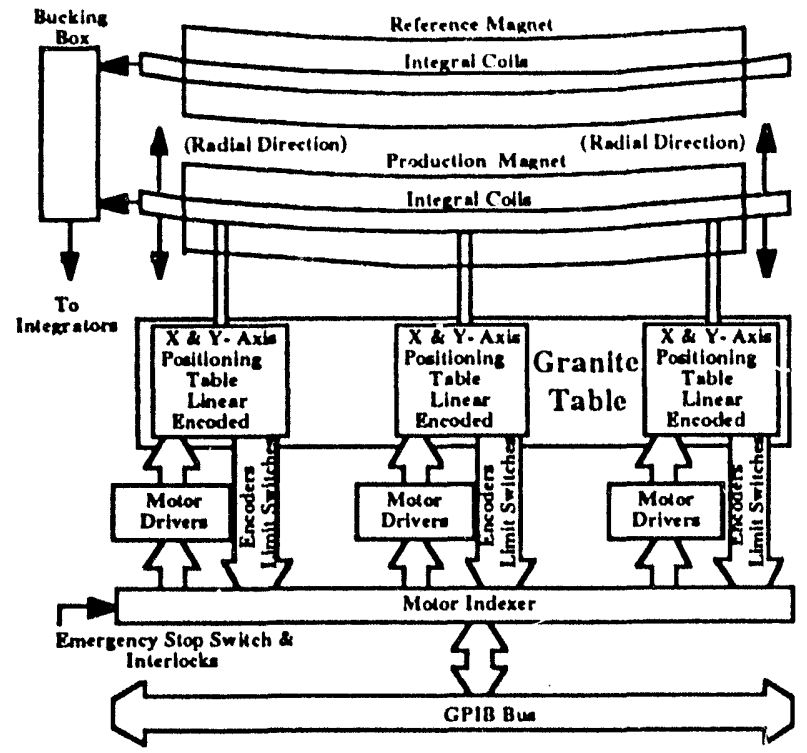

Fig. 1. Block diagram of the hardware assembly for the dipole measurement.

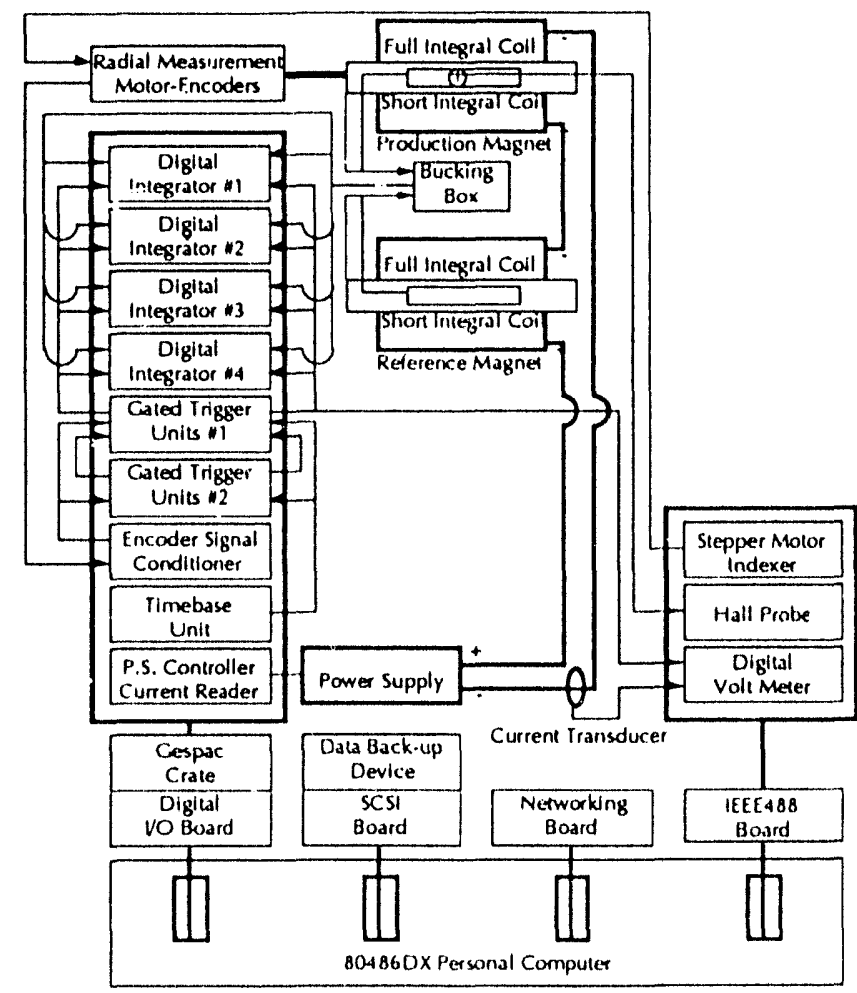

Fig. 2. Block diagram of the data acquisition system for the dipole measurement.

data are used for the calibration of the integral coil measurements.

Shown in Fig. 2. is a block diagram of the data acquisition system for the dipole measurements. Four high-precision digital integrators, which are voltage-to-frequency converters, are used: one each for the field integral and 2-D field of the reference magnet and two for the respective bucking signals between the two magnets. Since the integrator connections conform to G-64 Gespec Crate specifications, IBM digital I/O interfaces and other modules developed for measurement control and data acquisition were modified to configure to Gespec Crate requirements.

An operating system shell on MS-DOS called GPDAS has been developed to provide greater flexibility when taking measurements and performing data analyses [3]. The shell contains the software drivers to interface with GPIB and digital I/O boards, Gespec Crate, and other required PC boards.

The measurement procedures, including control of the magnet excitation current and data analyses in "real-time," are automated. During the prototype and production measurements of IS dipoles, it has been firmly established that relative accuracy and reproducibility of the field strength and field shape measurements are better than $1 \times 10^{-4}$.

\section{MULTIPOLE MEASUREMENT SYSTEMS}

Shown in Fig. 3 is a block diagram of the rotating coil system. A rotating coil cylinder, supported by two airbearings, is rotated by a DC motor. A 15 -bit absolute encoder triggers the integration of the induced voltage in the coil at 256 angular positions per turn. Two of the three systems are mudified versions of the rotating coil measurement bench developed at CERN and fabricated by Danfysik [4]. The third system, developed at ANL, has additional features for

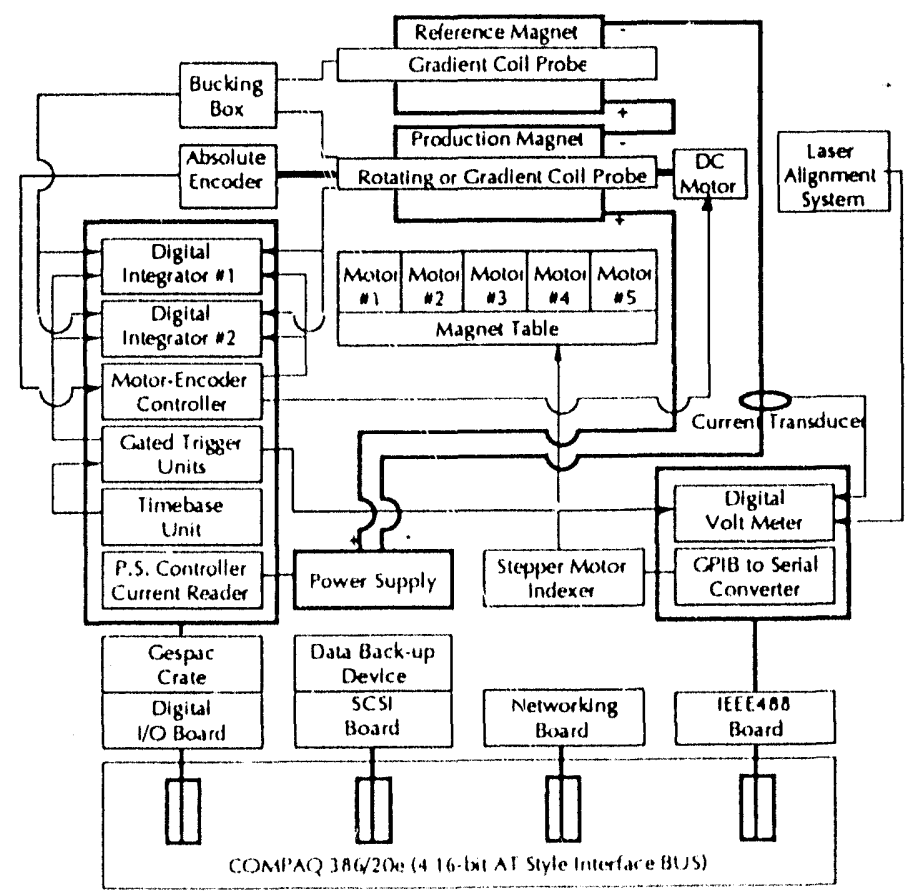

Fig. 3. Block diagram of the rewating coil system. 
measurement flexibilities such as adjusting the distance between the two air-bearings and the alignment of bore axis.

Since the air-bearing positions are fixed to the base table, the magnet position has to be controlled and pre-aligned to the rotating coil axis using five stepping motors. A precision laser and photo-quadrant detector are used for the alignment. The position of the laser, mounted on one end of the base table, is precisely measured from the axis of the rotating coil cylinder. The detector, mounted in a Taylor-Hopson ball, is placed on top of the testing magnet. From the harmonic analysis of the rotating coil measurements, the magnet is aligned to the rotating coil axis. Then, the detector reads the fiducial position with respect to the magnetic axis.

Prior to the above procedure, for SR quad measurement only, the bore axis is aligned to the air-bearing axis as in the following method. A second laser beam unit is installed and aligned with the air-bearing axis. The beam positions along the bore axis are detected by another photo-quadrant detector placed at the bore axis. Then, the quad is aligned by moving the bore axis to that of the laser beam. Since the rotating coil cylinder is supported by the two air-bearings, the coil rotates at the air-bearing axis. During the magnetic alignment, the magnet is moved only parallel to bore axis. This procedure not only ensures parallelism between the bore and magnetic axes within $\pm 0.2 \mathrm{mrad}$, but it also measures the offset of the two axes.

In addition to the rotating coil measurement, the fieldgradient integrals for the SR quads are also measured relative to the reference quad with a measurement reproducibility of \pm $2 \times 10^{-4}$. One "gradient-coil" probe in the reference quad is fixed along the bore axis. The second probe for the testing quad is installed along the axis of a G-10 cylinder. Since the measurement is conducted after the magnetic alignment, the probe in the testing quad is located along the magnetic axis.

\section{Rotating Coll Probes}

One of the critical components for the multipole measurements is the rotating coil probe. The cross section of a rotating coil geometry depicted in Fig. 4 has two different types of coils: "radial" and "tangential." The radial coil set is located in the plane of the $x$-axis, while the tangential coil set is installed on the cylinder surface. In Table 2 design parameters of the two types of coil sets for quad and sext measurements are listed. The ratio of the number of turns and the radii of the coils are denoted as $N_{a} / N_{b}$ and $R_{a} / R_{b}$, respectively. The coil paraneters, listed in Table 3 , are selected according to Table 2 for the SR quad and sext probes. The "short" tangential coils are used to measure the 2-D "body" field. $\triangle O$ is the coil angle relative to a reference.

The radial coil set consists of two separate coils, one for measuring the main field anc one for multipole coefficients after bucking the main field. The tangential coil set in Fig. 4

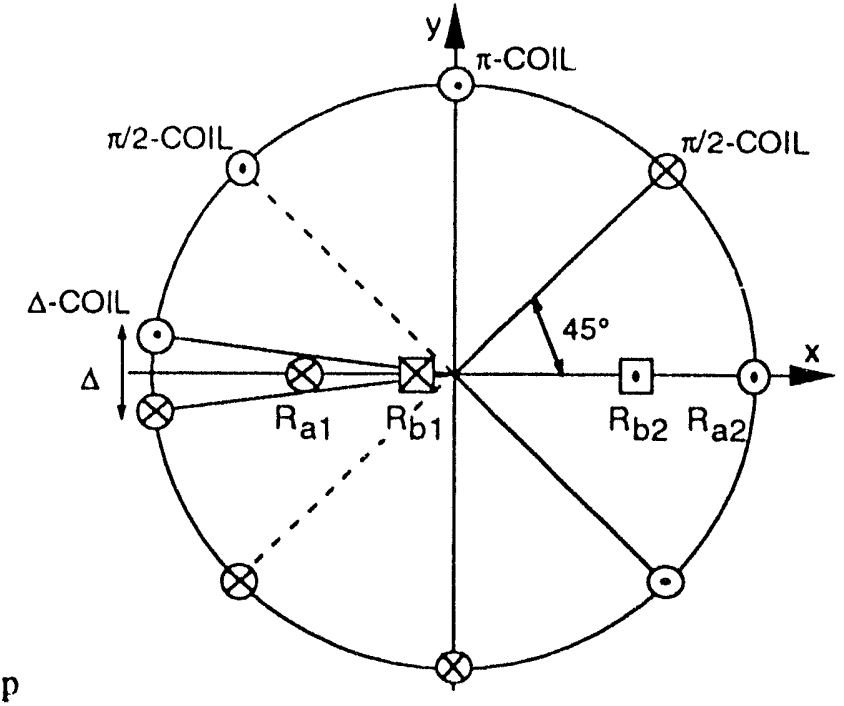

Fig. 4. Cross section of the radial and tangential coil geometries. The radial coil set is located on the $\mathrm{x}$-axis.

TABLE 2

DESIGN PARAMETERS OF ROTATING COIL PROBES

\begin{tabular}{ccc}
\hline Coil Parameter & Quad Coil & Sext Coil \\
\hline Badial Coil & & \\
$\mathrm{N}_{\mathrm{a}} / \mathrm{N}_{\mathrm{b}}$ & $1 / 2$ & $6 / 21$ \\
$\mathrm{R}_{\mathrm{a} 1} / \mathrm{R}_{\mathrm{a} 2}$ & 0.5 & 0.5 \\
$\mathrm{R}_{\mathrm{b} 2} / \mathrm{R}_{\mathrm{a} 2}$ & 0.625 & 0.6266 \\
$\mathrm{R}_{\mathrm{b} 1} / \mathrm{R}_{\mathrm{a} 2}$ & 0.125 & 0.4224 \\
& & \\
Tangencial Coil & & \\
$\mathrm{N}_{\mathrm{b}} / \mathrm{N}_{\Delta}$ & $1 / 6$ & $1 / 7$ \\
$\mathrm{~N}_{\mathrm{Q}} / \mathrm{N}_{\Delta}$ & $1 / 6$ & $2 / 7$ \\
$\mathrm{~N}_{\mathrm{S}} / \mathrm{N}_{\Delta}$ & $\ldots$ & $15.388^{\circ}$ \\
$\Delta \cdot \mathrm{Coil}^{\circ}$ & $19.471^{\circ}$ & \\
\hline
\end{tabular}

TABLE 3

ROTATING COIL PARAMETERS FOR SR QUAD AND SEXT MAGNET MEASUREMENTS

\begin{tabular}{|c|c|c|}
\hline Coil Parameter & Quad Coil & Sext Coil \\
\hline \multicolumn{3}{|l|}{ Radial Coil } \\
\hline $\mathrm{R}_{\mathrm{a} 2}(\mathrm{~mm})$ & 37.75 & 44.005 \\
\hline $\mathrm{N}_{\mathrm{a}}$ & 18 & $10+25 *$ \\
\hline$\Delta Q($ mrad $)$ & -1.597 & 2.20 \\
\hline \multicolumn{3}{|l|}{ Tangential Coil } \\
\hline $\mathrm{R}($ (nu) & 35.75 & 35.941 \\
\hline$N_{D}$ & 6 & ... \\
\hline \multirow[t]{2}{*}{$\mathrm{N}_{\mathrm{Q}}$} & & 6 \\
\hline & 3 & \\
\hline $\mathrm{N}_{S}$ & $\cdots$ & 6 \\
\hline $\mathrm{N}_{\Delta}$ & 36 & 21 \\
\hline$\Delta \emptyset$ (mrad), Full/Short & $2.19 / 0.4062$ & $.018 / 0.102$ \\
\hline Shon Coil lenght (m) & 0.406 & 0.102 \\
\hline
\end{tabular}

* lextra number of turns to increase the measurement sensitivity of the sext field serengith. 
consists of a " $\Delta$-coil," " $\pi$-coil," and two " $\pi / 2$-coils." The $\pi$ coil measures the dipole field component, while the two $\pi / 2$ coils measure the quad or sext field component, depending on the connection of the two coils. These coils are used for bucking the main field and also for measuring lower harmonics. The magnetic axes of quad and sext magnets are identified from the dipole and quad components, respectively.

The advantage of having two types of coil sets on the same cylinder is that the validity of the measurements can be crossexamined at the same condition. For the SR quad, for example, the measured relative sext coefficients from the two coils agree within $0.5 \times 10^{-4}$ at a radius of $25 \mathrm{~mm}$.

For the alignment parameters, however, the two coil sets behave some what differently. Shown in Fig. 5 are the stability of the laser beam and the magnetic axis of a SR quad measured for several hours. All the data, except for the positions of "Laser-2," were taken in a laboratory where the stability of the floor was not known. The laser beam, which is positioned on top of the quad, monitors the stability of the measurement system. From the data for "Laser-1," it is seen that the laser beam drifted more than $50 \mu \mathrm{m}$. The data for Laser-2, which were taken on a stable foor, drift less than 25 $\mu \mathrm{m}$. The measurements of the magnetic axis from the two coils agree to within $15 \mu \mathrm{m}$ and vary less than $10 \mu \mathrm{m}$ in both the $x$ - and $y$-axes.

Shown in Fig. 6 are the data of roll angle measurements for the quad and sext magnets. Except for the radial coil for quad (Rad-Q in Fig. 6), the roll angle measurements are repeatable within $\pm 0.1 \mathrm{mrad}$.

\section{CONCLUSIONS}

The relative measurement accuracy and reproducibility of the field integrals and field shapes for dipoles are better than $1 \times 10^{-4}$. The three rotating coil systems measure the integral and 2-D field strengths and multipole coefficients with relative accuracies of $\pm 2 \times 10^{-4}$. The measurement accuracies of the roll angle of the main field and the magnetic axis relative to the fiducial positions are better than $\pm 0.3 \mathrm{mrad}$ and $\pm 60 \mu \mathrm{m}$, respectively.

\section{REFERENCES}

(1) 7.Gev Advanced Photon Source, conceptual design report," Argonne Naional Laboratory, ANL-87-15, 1987.

[2] M. I. Green, "Fabrication and calculation of search coils," and L. Walckiers, "The harmonic-coil method," in CERN 92-05, 1992, edited by $S$. Turner.

[3] Y. Chung and K. Kim, "Development and application of general purpose data acquisition shell (GPDAS) at the Advanced Photon Source," Conf. Record of the 1991 IEEE Particle Acc. Conf., Vol. 2, pp. 1299-1301, 1991.

(4) O. Pagano, P. Rohmig, L. Walckiers and C. Wyss, "A highly automated measuring system for the LEP magnetic lenses," J. de Physique. Vol. 45. Colloque Cl, pp. 949-952, 1984.

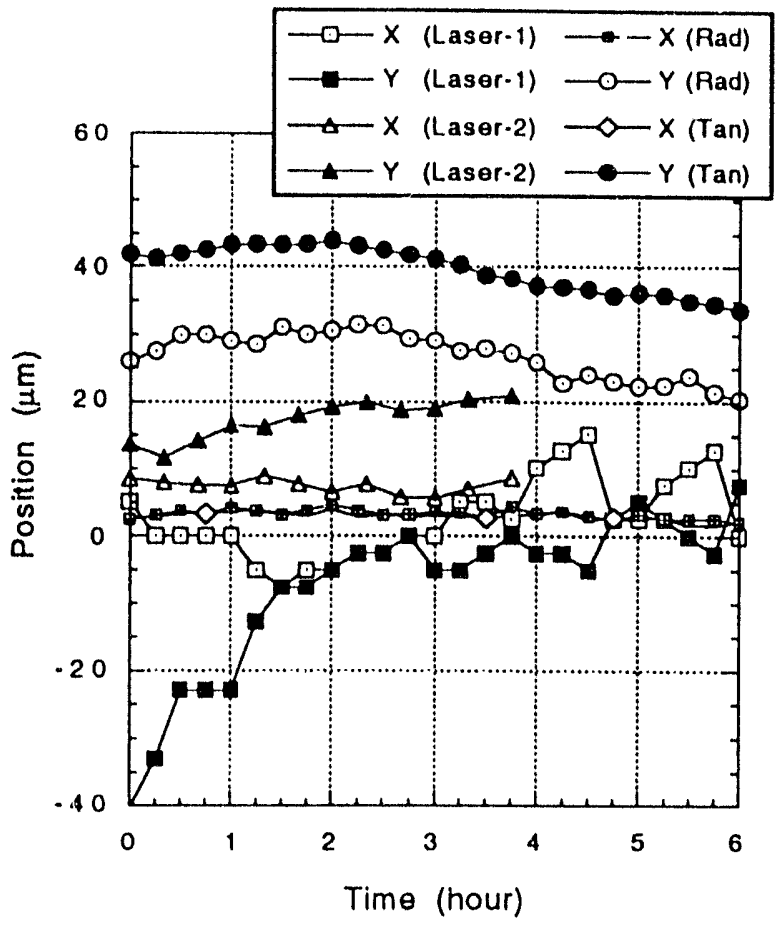

Fig. S. Stability of laser beam and magnetic axis measurements using radial (Rad) and tangential (Tan) coils. The data points for "Laser-1" were taken in a room with unspecified floor conditions, while those for "Laser-2" were taken in a stable and temperature-controlled room. The data for the laser have initial offsets.

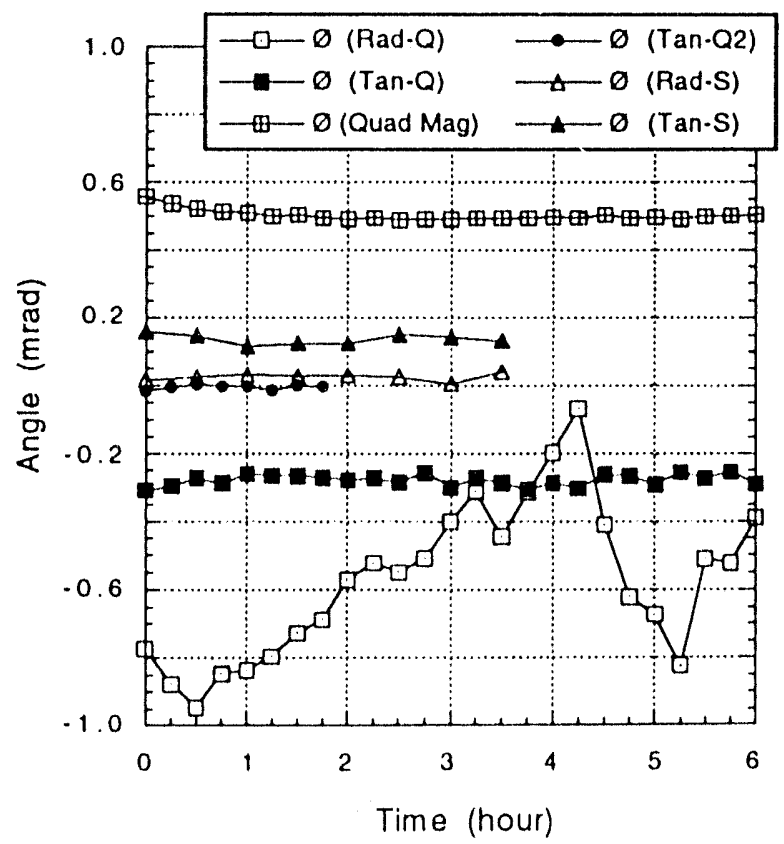

Fig. 6. Measurements of roll angles for the SR quad (Q) and sext (S) magnets using radial and tangential coils. The data for roll angle of the steel core (Quad Mag) have initial offset. 


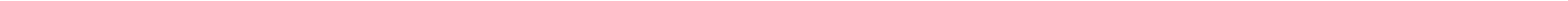


